\title{
PAEDiaTRIC NONBRONCHOSCOPIC BRONCHOALVEOLAR LAVAGE: OVERVIEW AND RECOMMENDATIONS FOR Clinical Practice
}

\begin{abstract}
NB-BAL is an effective procedure for the diagnosis of pulmonary disease processes in ventilated infants and children. This procedure is, however, not without risks to both patients and staff. Numerous complications of NB-BAL exist, with hypoxia being the most common. As a result, care should be taken in performing NB-BAL on haemodynamically unstable patients; patients with coagulation defects; and patients with cardiac or brain abnormalities.

This paper presents an overview of paediatric nonbronchoscopic bronchoalveolar lavage (NB-BAL) including: the rationale for $N B-B A L$; the complications associated with the procedure; indications and contraindications. It also recommends an evidence-based clinical guideline for performing the procedure in the paediatric

\section{Morrow BM, (PhD) $)^{1,2}$; Futter MJ (PhD) ${ }^{3}$; Argent AC (FCPaed, SA)}

Red Cross War Memorial Children's Hospital, Physiotherapy Department and Division of Associated Paediatric Disciplines, School of Child

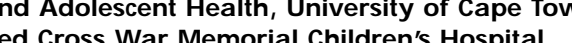
Paediatric Intensive Care Unit and Division of Critical adolescent Health, University of Cape Town.

ciences, University of Cape Town.
\end{abstract} intensive care unit.

By following the NB-BAL guidelines presented in this paper, one can ensure that an effective specimen is obtained from the lower respiratory tract, whilst minimising the risk to the patient.

KEY WORDS: NONBRONCHOSCOPIC BRONCHOALVEOLAR LAVAGE; PAEDIATRIC; INTENSIVE CARE; CLINICAL GUIDELINE.

\section{INTRODUCTION}

Diagnostic nonbronchoscopic bronchoalveolar lavage (NB-BAL) is a procedure performed by physiotherapists in paediatric intensive care units both locally (Morrow et al 2004a; Morrow and Argent 2001) and internationally (Burmester and Mok 2001). Despite this, there are currently no published NB-BAL protocols in physiotherapy journals.

This paper aims to present the rationale behind paediatric NB-BAL; the complications associated with the

CORRESPONDENCE TO:

Dr BM Morrow

Physiotherapy Department Red Cross War Memorial

Children's' Hospital Klipfontein Road Rondebosch, 7700

Tel: (021) $6585111(\mathrm{w})$ (021) $7050509(\mathrm{~h})$

Email: bmorrow@ich.uct.ac.za procedure; and it recommends an evidence-based NB-BAL protocol including indications, contraindications and technique, for use in the paediatric intensive care unit.

\section{RATIONALE}

Diagnosis of aetiological agents in childhood pneumonia is difficult as blood culture results are frequently negative (Grigg et al 1993). Tracheal aspirate cultures have relatively low sensitivity and are unreliable in most intubated patients due to contamination with organisms colonising the upper respiratory tract (Jourdain et al 1995).

Bronchoalveolar lavage (BAL) is an effective and reliable way of establishing the aetiology of pulmonary disease processes, in both adult and paediatric populations (Panero et al 1995, Koumbourlis and Kurland 1993, Alpert et al 1992). BAL is a far less invasive procedure than lung biopsy and appears to obtain comparable specimens from the lower respiratory tract (Bye et al 1987). 
Nonbronchoscopic bronchoalveolar lavage (NB-BAL) was first described in a paediatric population by Alpert et al (1992). It is a simple, effective, less time-consuming procedure that requires less expertise and is cheaper than bronchoscopic BAL (Prokop et al 1996, Pugin et al 1991, Minotuli et al 1990, Mann et al 1987, Caughey et al 1985). In addition, where there is diffuse disease, direct visualisation by bronchoscopy is not necessary. NB-BAL is able to sample fluid specifically from the lower respiratory tract, as demonstrated by the presence of alveolar macrophages in the lavage fluid. Diagnostic yields from $42 \%$ to $85 \%$ have been reported (Morrow and Argent 2001, Koumbourlis and Kurland 1993, Alpert et al 1992, Minotuli et al 1990, Piperno et al, 1988).

In addition to the diagnostic benefits, therapeutic benefits of NB-BAL have also been described. It was found to be effective in improving lung expansion in $84 \%$ of infants with radiological evidence of lobar collapse, which had not responded to conventional chest physiotherapy (Galvis et al, 1994). Dargaville et al (1999) noted that in three infants with meconium aspiration NB-BAL appeared to have a beneficial effect by removing meconium debris. Koumbourlis and Kurland (1993) also observed that two patients with atelectasis showed radiological evidence of improvement following NB-BAL.

\section{NB-BAL COMPLICATIONS:}

There are reports of nursing staff acquiring tuberculosis (TB) from children requiring endotracheal suctioning (Curtis et al 1999, Rabalais et al 1991), implying a potential risk of infection to the therapist performing NB-BAL. Appropriate infection control precautions should therefore always be taken, particularly in the South African context where infectious diseases such as TB are particularly rife (Zar et al 2005, Shingadia and Novelli 2003).

Early studies of paediatric NB-BAL reported no clinically significant patient complications (Schindler and Cox 1994, Koumbourlis and Kurland 1993, Alpert et al 1992, Minotuli et al 1990, Piperno et al 1988). The majority of these initial studies did not report oxygenation or ventilation requirements or document arterial blood gases. One cannot, therefore, conclude from them that NB-BAL is safe in extremely sick, unstable children and infants. Subsequent studies have highlighted potentially serious adverse effects of paediatric NB-BAL (Morrow et al 2004a, Morrow and Argent 2001, Burmester and Mok 2001). It must be noted that studies investigating the complications of paediatric NB-BAL have excluded patients with raised intracranial pressure, haemodynamic instability and coagulation disorders (Morrow et al 2004a, Morrow and Argent 2001) due to the potential complications identified from data relating to endotracheal suctioning (Darlow et al 1997, Alpert et al 1992, Singh et al 1991, Perlman and Volpe 1983, Anderson and Chandra 1976).

Hypoxia, which is a frequent complication of NB-BAL, is mild and selflimiting in most cases (Morrow et al 2004a, Burmester and Mok 2001, Morrow and Argent 2001, Dargaville et al 1999, Baughman and Conrado 1998). However, in some cases prolonged severe episodes of hypoxia have been reported, with the patient requiring escalation of oxygen and ventilatory support (Morrow et al 2004a, Morrow and Argent 2001, Burmester and Mok 2001). NB-BAL-induced hypoxia may be caused by a combination of: i) oxygen deprivation if the oxygen supply is disconnected; ii) interruption of ventilation with an associated decrease in airway pressure (Maggiore et al, 2003); iii) airway occlusion by the suction catheter (Morrow et al 2004a); iv) aspiration of intrapulmonic gas during prolonged suctioning (Ehrhart et al, 1981); v) the effect of the lavage itself (Ridling et al 2003, Burmester and Mok 2001); and vi) coughing.

During NB-BAL, the catheter remains in the tracheobronchial tree, thus partially obstructing the airway and markedly increasing airway resistance, for longer periods than during normal endotracheal suctioning. It is likely that the added airway obstruction caused by the suction catheter could also cause carbon dioxide retention. For the purposes of NB-BAL, repeated suction manoeuvres are performed without allowing recovery time. This repeated application of negative pressure may lead to loss of lung volume with resulting hypoxia (Morrow et al 2004a , Morrow et al 2004b, Simbruner et al 1981, Rosen and Hillard 1962). A large amount of saline is instilled into the lungs during NB-BAL. The volume of saline introduced into the lungs during the procedure may decrease the available surface area for gaseous exchange and unretrieved saline could interfere with alveolocapillary oxygen exchange (Ridling et al 2003). It is interesting to note that in many experimental models of acute lung injury, the injury is induced by means of saline lavage (Allen et al 2002, Rimensberger et al 1999, Neumann et al 1998).

Burmester and Mok (2001) were unable to identify predictive factors for NB-BAL complications. Dargaville et al (1999) were unable to demonstrate a propensity for desaturation based on severity of lung disease. In contrast to these studies, Morrow and Argent (2001) and Morrow et al (2004)a demonstrated that there is a significantly greater risk of more marked and more prolonged desaturation in patients with high oxygenation indices and low $\mathrm{PaO}_{2} / \mathrm{FiO}_{2}$ (partial pressure of oxygen / fraction of inspired oxygen) ratios, as seen in patients with acute respiratory distress syndrome and acute lung injury.

Cardiac arrhythmia with significant desaturation followed by death has been reported in a child undergoing bronchoscopic BAL with pulmonary hypertension and congestive heart failure secondary to upper airway obstruction (Wagener 1987). It was postulated that hypoxiainduced increased pulmonary vascular resistance could precipitate worsening heart failure. In addition, when there is pulmonary hypertension in the presence of high pulmonary vascular pressure, markedly negative intrapleural pressures during partially obstructed aspiration may result in fluid transudation from the pulmonary vessels thereby precipitating acute pulmonary oedema (Wagener 1987). Bye et al (1987) reported serious side effects of bronchoscopic BAL in two patients: one patient had a seizure and another experienced increased tachypnoea and low BAL fluid return and died 30 hours after the procedure. 
These authors mention the potential risk of fluid overload as a result of poor recovery of BAL fluid.

Prolonged, clinically significant changes in blood pressure requiring escalation of inotropic support have been reported following NB-BAL (Burmester and Mok 2001). Bronchial haemorrhage has been observed following nonbronchoscopic protected brush specimens and bronchoalveolar lavage (Morrow and Argent 2001, Labenne et al 1999). Diagnostic BAL in patients with pneumonia may cause intravascular translocation of toxins or mediators, producing pyrogenic and hypotensive effects (Pugin and Suter 1992, Labenne et al 1999).

Pneumothorax has been reported as a complication of NB-BAL in neonates (Labenne et al 1999). Pneumothorax is a particular risk of NB-BAL as the suction catheter is passed far beyond the ETT into the bronchi themselves (Anderson and Chandra 1976).

On application of negative suction pressure, most of the instilled saline is removed along with some of the alveolar lining fluid, which contains surfactant necessary for the patency and stability of the alveoli (Burmester and Mok 2001). This may result in atelectasis with subsequent hypoxia. As a result of the concern about washing out of surfactant it has been suggested that NB-BAL may exacerbate existing respiratory disease, particularly in infants with neonatal Respiratory Distress Syndrome. However, in a controlled trial, repeated NB-BAL in newborn infants did not appear to be associated with radiological changes suggesting that surfactant was not consistently removed (Kotecha 1999).

\section{INDICATIONS AND CONTRAINDICATIONS}

NB-BAL is effective in identifying pulmonary pathogenic processes (Morrow and Argent 2001, Kotecha 1999, Panero et al 1995, Koumbourlis and Kurland 1993, Minotuli et al 1990, Piperno et al 1988), and is a valuable diagnostic procedure for use in intubated, mechanically ventilated patients.

It is suggested that specific indications for NB-BAL should include radiological or clinical evidence of lung disease, raised infectious markers and a deteriorating clinical picture despite optimal management. It is felt that NB-BAL should ideally be performed on stable patients as soon after intubation as possible, before bacterial endotracheal tube colonisation has occurred and/or in the event of a changing clinical picture with signs of infection and unknown pathogen. The contraindications and precautions to NB-BAL, based on the complications discussed previously, are listed in the insert below.

Considering the high diagnostic yield of NB-BAL, it may be worth the risk of performing the procedure in certain high-risk patients despite the presence of contraindications, where there have been no other positive cultures. These patients, however, should be selected carefully. Consideration should be given to the risk: benefit ratio for the child, the financial cost of the investigations, and how NB-BAL findings would influence patient management.

It is recommended that special precautions be taken with regard to sedation, level of preoxygenation and technique during NB-BAL in patients with high oxygenation and ventilation requirements, particularly in patients with oxygenation indices $>10$, ventilation indices $>20$ and with $\mathrm{PaO}_{2} / \mathrm{FiO}_{2}<150$ (Morrow and Argent 2001).

Bronchoscopic BAL (which allows direct visualisation of the airways) is indicated where there is localised lung disease and ETT $>4,5 \mathrm{~mm}$ internal diameter. This is preferable as specific sampling from the area of pathology can be obtained. In infants with small ETT internal diameters, and unilateral lung disease, directed blind NB-BAL can be performed by positioning the patients with their head away from abnormal lung (Kotecha 1999).

The potential complications of NB-BAL should not be minimised. All patients should be carefully monitored throughout the procedure (Pattishall et al 1988) and resuscitation equipment should be available at all times.

\section{PAEDIATRIC NB-BAL TECHNIQUE}

Numerous NB-BAL techniques have been reported (Burmester and Mok 2001, Dargaville et al 1999, Koumbourlis and Kurland 1993, Alpert et al 1992). Based on these, and other studies, Morrow et al (2004a) describe a simple, effective NB-BAL technique which maintains ventilation throughout the procedure and effectively reduces the number of complications, specifically that of desaturation. This method is effective, inexpensive, and easy to perform and is therefore the recommended NB-BAL technique. A detailed description of the technique is presented in the series of boxed inserts:

\section{CONTRAINDICATIONS OR PRECAUTIONS TO PAEDIATRIC NB-BAL}

- Haemodynamic instability

- Pulmonary haemorrhage.

- Pulmonary oedema.

Cor pulmonale with pulmonary hypertension.

Raised intracranial pressure.

Congestive cardiac failure.

- Coagulopathy, with platelet count $<50 \times 10^{9} / 1$. NB-BAL may be considered after transfusion of blood products if the coagulopathy has resolved.

- Neonatal respiratory distress syndrome, due to the concern about washing out of surfactant.

- Premature, small-for-gestational-age infants due to the risk of intraventricular haemorrhage.

- NB-BAL is not appropriate after a decision has been made to withdraw ventilatory support, offer palliative care or limit invasive and unpleasant procedures. 
1.Prior to NB-BAL, patients should receive sedation - usually morphine or midazolam as a $0,1 \mathrm{mg} / \mathrm{kg}$ intravenous push. In some cases additional muscle paralysis is required.

2. Electrocardiographical and pulse oximetry monitoring must be used throughout.

3. A manual resuscitation bag, with oxygen flow turned on, should be ready at the patient's side.

4. Preoxygenate with $100 \%$ inspired oxygen for approximately one minute prior to NB-BAL and maintain this $\mathrm{FiO}_{2}$ throughout until the patient's oxyhaemoglobin saturation $\left(\mathrm{SaO}_{2}\right)$ returns to prelavage levels.

5. Remove pressure probe or seal at ventilator port and place two layers of Tegaderm transparent dressing (3M Health Care, USA) over the aperture to seal the ventilator system. Pierce the Tegaderm with a needle.

\section{NB-BAL PROCEDURE}

1. Pass a suction catheter of appropriate size through the Tegaderm, into the ETT; wedge the catheter as far distally as possible and then withdraw very slightly $(<0,5 \mathrm{~cm})$. The catheter should not have side-holes in order to facilitate delivery of saline to the distal air spaces beyond the point of wedging and to minimise mucosal trauma caused by the adherence of the side-holes to the bronchial walls (Morrow et al 2004a , Dargaville et al 1999).

2. Introduce the first lavage volume of $1 \mathrm{ml}$ per $\mathrm{kg}$ body weight of $0,9 \%$ saline at room temperature, through the catheter. It has been suggested that by adjusting BAL volume to body weight, a constant fraction of epithelial lining can be obtained (Ratjen and Bruch 1996).

3. Attach the suction catheter to a mucus extractor with wall unit suction applied. One obtains a better fluid return with continuous pump suction than with intermittent manual aspiration (Caughey et al 1985).

4. Continue suctioning until no more saline is withdrawn. Movement of the catheter of about $5 \mathrm{~mm}$ is permissible to maximise fluid return.

5 . Two further lavages of $1 \mathrm{ml} / \mathrm{kg}$ body weight of saline solution are performed through the same catheter in the same position. The catheter is not withdrawn in between suction events.

6. The first aliquot is discarded, and the subsequent two are collected in the same mucus extractor and sent for analysis (Ratjen and Bruch 1996, Grigg et al 1993). The cell profile of the first aliquot differs from the subsequent two, containing mainly bronchial cellular material and epithelial cells. Separation of the first portion of the aspirated fluid may thus improve information gained about lower respiratory tract pathology (Pohunek et al 1996).

7.If a patient's $\mathrm{SaO}_{2}$ drops to $<81 \%$ at any stage during the procedure the catheter should be withdrawn immediately while attempting to suction as much fluid back as possible in the process. If immediate improvement does not occur, the patient should be manually ventilated with $100 \%$ oxygen until $\mathrm{SaO}_{2}$ returns to pre-lavage levels (Morrow et al 2004a) .

\section{PNB-BAL PROCESSING}

1. The second and third aliquot together are sent to the laboratory.

2. According to clinical indications, the specimen may be sent for microscopy, culture and sensitivity; viral culture; TB studies; extended fungal culture; Pneumocystis jiroveci immunofluorescence; and histology for any or all of cytology, fat laden macrophages, haemosiderin laden macrophages and viral inclusion bodies.

\section{CONCLUSION:}

This paper presents an overview of the reasons for performing diagnostic NB-BAL; the complications associated with the procedure; and it recommends an evidence-based NB-BAL protocol for use in the paediatric intensive care unit.

NB-BAL is an effective procedure for the diagnosis of pulmonary disease processes in ventilated infants and children. This procedure is, however, not without risks to both patients and staff. Numerous complications of NB-BAL exist, with hypoxia being the most common. As a result, care should be taken in performing NB-BAL on haemodynamically unstable patients and patients with coagulation defects, cardiac or brain abnormalities.

By following a defined protocol, one can ensure that the most effective specimen is obtained from the lower respiratory tract, whilst minimising the risk to the patient.

\section{REFERENCES}

Alpert BE, O'Sullivan BP, Panitch HB 1992 Nonbronchoscopic Approach to Bronchoalveolar Lavage in Children with Artificial Airways. Pediatric Pulmonology 13: 38-41.

Allen G, Lundblad LKA, Parsons P, Bates JHT 2002 Transient mechanical benefits of a deep inflation in the injured mouse lung. Journal of Applied Physiology 93:1709-1715

Anderson K, Chandra K 1976 Pneumothorax secondary to perforation of sequential bronchi by suction catheters. Journal of Pediatric Surgery 11:687-693

Baughman RP, Conrado CE 1998 Diagnosis of Lower Respiratory Tract Infections. What We Have and What Would Be Nice. Chest 113 Suppl: 219S-223S.

Bradshaw D, Bourne D, Nannan N 2003 What are the leading causes of death among South African children? MRC Policy Brief No. 3, December

Burmester M, Mok Q 2001 How safe is non-bronchoscopic bronchoalveolar 
lavage in critically ill mechanically ventilated children? Intensive Care Medicine 27:716-721

Bye MR, Bernstein L, Shah K, Ellaurie M, Rubenstein A 1987 Diagnostic bronchoalveolar lavage in children with AIDS. Pediatric Pulmonology 3: 425-428

Caughey G, Wong H, Gamsu G, Golden J 1985 Non-bronchoscopic bronchoalveolar lavage for the diagnosis of Pneumocystis carinii pneumonia in the acquired immunodeficiency syndrome. Chest 88: 659-662.

Curtis AB, Ridzon R, Vogel R, McDonough S, Hargreaves J, Ferry J, Valway S, Onorato IM 1999 Extensive transmission of Mycobacterium tuberculosis from a child. New England Journal of Medicine 341: 1491-1495.

Dargaville PA, South M, McDougall PN 1999 Comparison of Two methods of Diagnostic Lung Lavage in Ventilated Infants with Lung Disease. American Journal of Respiratory and Critical Care Medicine 160: 771-777.

Darlow BA, Sluis KB, Inder TE, Winterbourn CC 1997 Endotracheal Suctioning of the Neonate: Comparison of Two Methods as a Source of Mucus Material for Research. Pediatric Pulmonology 23: 217-221.

Ehrhart IC, Hofman WF, Loveland SR 1981 Effects of endotracheal suction versus apnea during interruption of intermittent or continuous positive pressure ventilation. Critical Care Medicine 9 (6): 464-468.

Galvis AG, Reyes G, Nelson WB 1994 Bedside Management of Lung Collapse in Children on Mechanical Ventilation: Saline Lavage - Simulated Cough Techniques Proves Simple, Effective. Pediatric Pulmonology 17:326-330.

Grigg J, Van den Borre C, Malfroot A, Pierard D, Wang D, Dab I 1993 Bilateral fiberoptic bronchoalveolar lavage in acute unilateral lobar pneumonia. Journal of Pediatrics 122: 606-608.

Jourdain B, Novara A, Joly-Guillou ML, Dombret MC, Calvat S, Trouillet JL, Gibert C, Chastre J 1995 Role of quanti- tative cultures of endotracheal aspirates in the diagnosis of nosocomial pneumonia. American Journal of Respiratory and Critical Care Medicine 152: 241-6.

Koumbourlis AC, Kurland G 1993 Nonbronchoscopic Bronchoalveolar Lavage In Mechanically Ventilated Infants: Technique, Efficacy, and Applications. Pediatric Pulmonology 15: 257-262.

Kotecha S 1999 Bronchoalveolar Lavage of Newborn Infants. Pediatric Pulmonology Suppl. 18: 122 - 124.

Labenne M, Poyart C, Rambaud C, Goldfarb B, Pron B, Jouvet P, Delamare C, Sebag G, Hubert P 1999 Blind protected specimen brush and bronchoalveolar lavage in ventilated children. Critical Care Medicine 27: 2537-2543

Maggiore SM, Jonson B, Richard J-C, Jaber S, Lemaire F, Brochard L (2001) Alveolar Derecruitment at Decremental Positive End-Expiratory Pressure Levels in Acute Lung Injury. American Journal of Respiratory and Critical Care Medicine 164: 795-801.

Mann JM, Altus CS, Webber CA, Smith PR, Muto R, Heurig AE 1987 Nonbronchoscopic lung lavage for diagnosis of opportunistic infection in AIDS. Chest 91: 319-322.

Minotuli R, Eden E, Brachfeld C 1990 Bronchoalveolar lavage via a modified stomach tube in intubated patients with the acquired immunodeficiency syndrome and diffuse pneumonia. Thorax 45: 771-773

Morrow B, Argent A 2001 The risks and complications of non-bronchoscopic bronchoalveolar lavage in a paediatric intensive care unit. Pediatric Pulmonology 32: 378 - 384.

aMorrow B, Futter M, Argent A 2004 A simple method of reducing the complications of paediatric nonbronchoscopic bronchoalveolar lavage. Pediatric Pulmonology 38: 217-221.

bMorrow B, Futter M, Argent A 2004 Endotracheal suctioning: from principles to practice. Intensive Care Medicine 30: $1167-1174$
Neumann P, Berglund JE, Mondéjar EF, Magnussen A, Hedenstierna G 1998 Dynamics of lung collapse and rerecruitment during prolonged breathing in porcine lung injury. Journal of Applied Physiology 85:1533-1543.

Pattishall EN, Noyes BE, Orenstein DM 1988 Use of bronchoalveolar lavage in immunocompromised children with pneumonia. Pediatric Pulmonology 5:1-5.

Panero A, Roggini M, Moretti C, Contini C, Bucci G 1995 Pneumocystis carinii pneumonia in preterm infants: report of two cases successfully diagnosed by non-bronchoscopic bronchoalveolar lavage Acta Paediatrica 84:1309 - 1311.

Perlman JM, Volpe JJ 1983 Suctioning in the Preterm Infant: Effects on Cerebral Blood Flow Velocity; Intracranial Pressure, and Arterial Blood Pressure. Pediatrics 72: 329-334.

Piperno D, Gaussorgues P, Bachmann P, Jabouley JM, Robert D 1988 Diagnostic value of non-bronchoscopic bronchoalveolar lavage during mechanical ventilation. Chest 93: 223.

Pohunek P, Pokorna H, Striz I 1996 Comparison of cell profiles in separately evaluated fractions of broncho-alveolar lavage (BAL) fluid in children. Thorax 51(6): 615-618.

Prokop A, Gawenda M, Krüger I, Pichlmaier H 1996 Value of Bronchoscopic Pneumonia Diagnosis: Prospective Study. World Journal of Surgery 20: $22-26$.

Pugin J, Auckenthaler R, Mili N, Janssens JP, Lew PD, Suter PM 1991 Diagnosis of ventilator-associated pneumonia by bacteriologic analysis of bronchoscopic and nonbronchoscopic 'blind' bronchoalveolar lavage fluid. American Review Respiratory Diseases 143:1121-1129.

Pugin J, Suter PM 1992 Diagnostic bronchoalveolar lavage in pateints with pneumonia produces sepsis-like systemic effects. Intensive Care Medicine 18: $6-10$ 
Rabalais G, Adams G, Stover B 1991 PPD skin test conversion in health care workers after exposure to Mycobacterium tuberculosis infection in infants. Lancet 338: 826.

Ratjen F, Bruch J 1996 Adjustment of Bronchoalveolar Lavage Volume to Body Weight in Children. Pediatric Pulmonology 21:184-188.

Ridling DA, Martin LD, Bratton SL 2003 Endotracheal Suctioning With or Without Instillation of Isotonic Sodium Chloride Solution in Critically Ill Children. American Journal of Critical Care 12: 212 - 219

Rimensberger PC, Cox PN, Frndova H, Bryan AC 1999 The open lung during small tidal volume ventilation: Concepts of recruitment and "optimal" positive end-expiratory pressure. Critical Care Medicine 27:1946-1952

Rosen M, Hillard EK 1962. The effects of negative pressure during tracheal suction. Anesthesia and Analgesia... Current Researches 41: 50 - 57.

Schindler MB, Cox PN 1994 A Simple Method of Bronchoalveolar Lavage. Anaesthetic Intensive Care 22:66-68.

Shingadia D, Novelli V 2003 Diagnosis and treatment of tuberculosis in children. Lancet Infect Dis 3: 624-632.

Simbruner G, Coradello H, Foder M, Havelec L, Lubec G, Pollak A 1981 Effect of tracheal suction on oxygenation, circulation, and lung mechanics in newborn infants. Archives of Diseases in Childhood 56: 326 - 330.
Singh NC, Kissoon N, Frewen T, Tiffin N 1991 Physiological responses to endotracheal and oral suctioning in paediatric patients: the influence of endotracheal tube sizes and suction pressures. Clinical Intensive Care 2: 345-350.

Wagener JS 1987 Fatality following fiberoptic bronchoscopy in a two year old child. Pediatric Pulmonology 3: 197-199.

Zar HJ, Hanslo D, Apolles P, Swingler G, Hussey G 2005 Induced sputum versus gastric lavage for microbiological confirmation of pulmonary tuberculosis in infants and young children: a prospective study. Lancet 365: 130-134

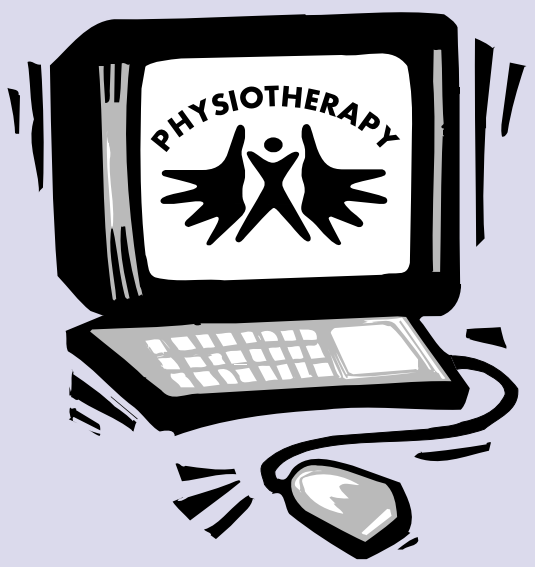

CHECK OUT THE SASP'S W EBSITE AT w w w.physiosa.org.za
TO ADVERTISE IN THE SOUTH AFRICAN J OURNAL OF PHYSIOTHERAPY, CONTACT AM ERICO AT SASP HEAD OFFICE: Tel: (011) 485-1467, Fax: (011) 485-1613

E-mail: pr@saphysio.co.za

A RTICLES FOR THE SOUTH AFRICAN

J OURNAL OF PHYSIOTHERAPY:

$A$ II articles $m$ ust be submitted

in an electronic format directly

to the Editor:

Prof CJ Eales,

P.O. Box 356 M ossel Bay 6500 Email: eales@icon.co.za 